\title{
Calidad del agua de unidades odlontológieas
}

\author{
Sara Ávila de Navia ${ }^{1}$, Sandra Mónica Estupiñán Torres ${ }^{1}$, Diana Milena Estupiñán Torres²
}

1 Programa de Bacteriología y Laboratorio Clínico. Universidad Colegio Mayor de Cundinamarca. Bogotá, Colombia. Suramérica.

2 Facultad de Odontología. Universidad Antonio Nariño. Villavicencio - Meta, Colombia. Suramérica.

Correspondencia: sestupinan@unicolmayor.edu.co

\section{RESUMEN}

El agua es una fuente importante de contaminación en pacientes odontológicos, esta contaminación se ha clasificado en dos clases: la primera, proveniente del suministro primario de agua, es decir, del agua pública y, la segunda, la que se encuentra adherida a las paredes de las mangueras de la unidad odontológica formando una biocapa, que se forma por los fluidos orales que son aspirados durante el procedimiento. La biocapa estará continuamente contaminando las mangueras de la unidad y expulsando microorganismos a la cavidad oral del paciente donde se generan aerosoles que pueden contaminar el ambiente, las superficies, los instrumentos y al personal de salud, lo que representa un riesgo en salud pública. En Colombia, no existen reportes oficiales ni datos que permitan controlar este problema de salud pública, por lo que los riesgos de contaminación e infección bacteriana siguen siendo latentes en la actualidad.

Palabras clave: odontología, infecciones oportunistas, microbiología del agua, salud pública.

\section{Water quality of dental units}

\section{ABSTRACT}

Water is a main source of contamination in dental patients, classified into two classes: the first from the primary water supply, (ie,public water) and the second one, which is attached to the hoses walls of the dental unit, forming a biofilm. Due to stagnation of oral fluids are aspirated during the procedure.

The biofilm will be continuously polluting the hoses from the unit and expelling microorganisms to the patient's oral cavity where they generate aerosols that can contaminate the environment, surfaces, instruments and health personnel, which is a public health risk. In Colombia there are not official reports or data to control this public health problem, so the risk of bacterial contamination and infection are still latent today.

Keywords: odontology, opportunistic infections, public health, water contamination, water microbiology. 


\section{INTRODUCCIÓN}

En los consultorios odontológicos los instrumentos manuales y los conductos de la unidad odontológica están siempre en contacto con el agua, lo cual genera un ambiente propicio para el desarrollo de microorganismos. Por esta razón es importante establecer la calidad microbiana del agua, con el fin de reducir el riesgo de contaminación, ya que ésta entra en contacto directo con el paciente y con el personal de salud y puede ocasionar enfermedades bajo condiciones especiales o en personas inmunocomprometidas $(1,2)$.

La cantidad de unidades formadoras de colonia (UFC) en el agua usada para enfriar o irrigar durante tratamientos odontológicos no quirúrgicos debe ser la mínima posible, teniendo en cuenta los parámetros establecidos para el agua potable. La decisión de emplear agua estéril durante estos tratamientos debe basarse en la complejidad del procedimiento, el estado inmunológico del paciente y otros factores de riesgo potencial para infecciones como la endocarditis bacteriana (3).

El agua empleada en tratamientos odontológicos debe contener recuentos de colonias tan bajas como sea posible. Las guías de calidad de la Asociación Dental Americana (ADA), en lo que se refiere a los conductos de agua en las unidades de odontología, proponen una meta de 200 $\mathrm{UFC} / \mathrm{mL}$ de bacterias aerobias mesofílicas heterotróficas. Se ha informado niveles de contaminación en el agua para tratamiento dental que exceden 1.000.000.000 UFC/mL La mayoría de los organismos recuperados de las líneas de agua en la unidad odontológica son naturales en ambientes acuáticos y se han reportado bacterias con un posible origen humano (4).
Aunque la fuente de microorganismos que provocan enfermedades infecciosas en la comunidad no siempre se identifica, en la práctica odontológica se han descrito sólo algunas infecciones probablemente debido a las escasas mediciones de microorganismos en el agua de las unidades odontológicas. En el 2007 se reportó el caso de una infección ocular que se inició por el contacto con un chorro de agua proveniente de la pieza de mano (5). Una publicación de 1987 de la Revista Dental Británica presentó dos casos de pacientes infectados con Pseudomonas aeruginosa en una clínica odontológica. En 1994, se registró la muerte de un odontólogo debido a una neumonía causada por Legionella pneumophila y la infección fue atribuida a la inhalación del patógeno durante el uso de los instrumentos odontológicos (6). Recientemente, un estudio llevado a cabo en el Reino Unido, sugiere que puede haber asociación entre el asma y la exposición ocupacional a líneas de agua de las unidades odontológicas contaminadas (7).

Las metas para la prevención de las enfermedades infecciosas son eliminar o reducir la exposición ante microorganismos, lo que también se conoce como control de la infección. Teniendo en cuenta que la odontología es un líder en el campo de la atención a la salud en el establecimiento y mantenimiento del control de las infecciones estas consideraciones son importantes $(8,9)$.

\section{Fuentes de contaminación en unidades odontológicas}

El equipamiento necesario de una unidad odontológica incluye el sistema de aspiración quirúrgico, equipo dental con módulos para turbina, micromotor y jeringa con funcionamiento de agua, aire y spray. Este equipamiento está conectado mediante un sistema central de tuberías generalmente 
de plástico que proceden de un depósito de agua (10). Estas líneas de agua son conductos de pequeño calibre que se emplean para activar o enfriar los instrumentos dentro de la cavidad oral. El agua de estos tubos crea las condiciones para el crecimiento de bacterias, lo que determina que tanto los profesionales como los pacientes estén expuestos al riesgo de infección (11).

El empleo de este equipamiento, sobre todo el de los micromotores de alta velocidad, generan gran cantidad de aerosoles, y si el agua de la unidad odontológica está contaminada, puede afectar el área de trabajo, con el consiguiente riesgo laboral y para el paciente (10). Las piezas de mano se contaminan internamente y el material retenido en el interior puede ser desalojado durante varias horas de operación. Es decir, estos instrumentos que frecuentemente succionan saliva $y$ sangre, pueden contaminarse en la boca de un paciente y arrojar material orgánico, potencialmente infeccioso, en las bocas de otros pacientes (12).

Se recomienda la esterilización de las piezas manuales en autoclave entre un paciente y otro; pero como la mayoría de piezas manuales y ángulos de profilaxis no resisten altas temperaturas, ni pueden tratarse con desinfectantes químicos, su tratamiento consiste principalmente en una limpieza externa con una solución desinfectante y una limpieza interna haciendo funcionar la pieza y descargar el agua en un vertedero por 20 ó 30 segundos. Este tipo de tratamiento reduce pero no elimina el problema de transmisión de la infección a partir de la unidad odontológica (13-15).

La principal fuente de agentes bacterianos que permite el desarrollo de biopelículas en los sistemas de agua de las unidades dentales, parece ser los abastecimientos de agua local o municipal que generalmente proporcionan agua potable con niveles bajos de bacterias saprófitas. Otra posible fuente de contaminación es la aspiración de la saliva del paciente contaminada con bacterias. Esta situación, en los países más avanzados, ha sido controlada por la utilización de unidades odontológicas modernas equipadas con sistemas de válvulas antireflujo $(16,17)$. Una de las características de los conductos de agua de las unidades odontológicas es su propensión a crear rápidamente biopelículas en las paredes de los conductos plásticos que llevan el agua hacia las piezas de mano, a los raspadores sónicos, a los ultrasónicos y a las jeringas de aire-agua usadas en el tratamiento de los pacientes (1).

Un "biofilm" o biopelícula es una agrupación de bacterias y otros microorganismos que segregan matrices poliméricas que les protegen del exterior, formando una capa muy fina que les ayuda a superar condiciones adversas. Estructuralmente tienen poros que permiten el paso de nutrientes a los microorganismos en dicha colonia, con lo cual se facilita la producción de polisacáridos que protegen a las células de cualquier agresión. Los microorganismos localizados en la parte más externa de la película, así como fragmentos de ésta, pueden ser arrastrados por el flujo de agua, contaminando los sistemas de irrigación en las unidades dentales $(8,18)$.

Los factores que favorecen esta colonización principalmente son: a.) El pequeño diámetro de la jeringa triple (1/8 a $1 / 16$ pulgadas) y su gran relación área-volumen, que en asociación a la baja presión de agua y poco flujo utilizado en los procedimientos odontológicos, facilita la acumulación de 
bacterias procedentes del sistema de distribución de agua potable. b.) El uso de agua caliente a una temperatura cercana a la corporal, que facilita el crecimiento de bacterias. c.) La utilización de filtros, con el objetivo de remover partículas provenientes del agua pública no tiene ningún efecto sobre las bacterias y lo único que logra es disminuir el flujo del agua, facilitando la colonización (4).

La formación de biopelículas en cualquier sistema de conducción de agua puede favorecer el crecimiento de patógenos oportunistas y aumentar sus concentraciones (19). Así, por ejemplo, estudios realizados reflejan que un $24 \%$ de agua de estaciones odontológicas, presentan altas concentraciones de $P$. aeruginosa y de Legionella spp. La presencia de estos microorganismos se puede ver favorecida por el hallazgo de amebas en los biofilms, las cuales son el principal hospedador de Legionella spp. y otras bacterias del género Pseudomonas (8, 20-22). Se ha demostrado que la concentración de especies correspondientes al género Mycobacterium, incluyendo Mycobacterium chelonae y Mycobacterium gordonae en las unidades dentales alcanza valores 400 veces superiores a los encontrados en aguas potables $(8,23,24)$.

Se han utilizado diferentes estrategias para evitar la colonización de los conductos de las unidades odontológicas, una de estas es el uso de agentes químicos, que reducen la cantidad de microorganismos pero no destruyen la biopelícula aun cuando estos se apliquen periódicamente (25).

El dióxido de cloro, es un biocida que previene la corrosión del metal, y se ha demostrado que permite reducir el recuento bacteriano a menos de $200 \mathrm{UFC} / \mathrm{mL}$ (26).
El paso de agua por conductos estimulados por una pequeña corriente eléctrica, ocasiona destrucción o mala formación del biofilm y se altera la morfología bacteriana, además el número de bacterias disminuyó la primera semana, y presentó un aumento gradual posteriormente (27).

Otra estrategia, ha sido el uso de unidades con un sistema de reservorio de agua independiente, en el que se adiciona agua desionizada, en el Reino Unido se evaluó si el uso de este sistema reducía los recuentos microbianos, se concluyó que los recuentos disminuyeron en las unidades que contaban con este sistema independiente, se recomendó el monitoreo rutinario de la efectividad de éste sistema (28).

\section{Bacterias presentesen el agua de las unidades dentales}

El objetivo principal de los trabajadores de la salud es reducir al máximo el riesgo de contaminación con microorganismos que puedan estar presentes en todos los instrumentos utilizados en los diferentes procedimientos, así como en el agua. Sin embargo, hay ocasiones en que esto no es posible, puesto que diferentes objetos, por su uso continúo o por su manipulación, facilitan la colonización de bacterias que en muchos casos pueden ser patógenas para la salud de los usuarios y del mismo personal de salud. Así mismo, el riesgo de contagio paciente-odontólogo-paciente es alto $(10,29,30)$.

En el caso del agua utilizada en las unidades odontológicas, se han encontrado diversos tipos de bacterias gracias a estudios bacteriológicos realizados. Aunque en muchas ocasiones los microorganismos hallados no son patógenos puesto que algunos provienen directamente de la flora normal bucal, otros provienen directamente 
del agua o de la biocapa que se forma en la superficie de las jeringas dentales por su constante exposición a la humedad. Los microorganismos dentro de los conductos acuáticos de las unidades odontológicas existen en 2 tipos de comunidades: uno está presente en el agua y se le conoce como microbio planctónico, el otro está en forma de sésil unido a las paredes interiores de los conductos acuáticos denominado biocapa o biopelicula (8).

En 1971 se dio a conocer el primer informe de microbios en el agua, que provino del estudio de 10 unidades odontológicas en tres clínicas privadas del área de San Francisco, Estados Unidos, reportando una concentración promedio de $180.000 \mathrm{UFC} / \mathrm{mL}$. En contraste a esto, el agua potable de los consultorios mostró un promedio de $15 \mathrm{UFC} /$ mL (23). En 1976, dos clínicas odontológicas del ejército informaron tener entre 200.000 y $500.000 \mathrm{UFC} / \mathrm{mL}$ en los instrumentos manuales, en las jeringuillas de aire/agua y en los conductos de los contadores ultrasónicos (31). En un estudio realizado en 1975 en Noruega, se demostró la presencia de bacterias en el agua contenida en las unidades odontológicas, en particular en los equipos de las facultades de odontología (32). El agua de las unidades odontológicas de dos unidades que participaron en un estudio que se realizó en 1984 en Chicago produjo conteos de hasta 50.00o UFC/mL (33).

Un estudio más reciente sobre el agua de las unidades odontológicas de los Estados Unidos reveló que el $72 \%$ de 150 unidades en 54 lugares de Washington, Oregon y California contenían elevados niveles de bacterias con promedios de $49.700 \mathrm{UFC} / \mathrm{mL}$ en los conductos de las jeringuillas de aire-agua y \}ría de las bacterias detectadas son de una muy baja patogenicidad o son patógenos oportunistas que provocan enfermedad sólo bajo condiciones especiales o en personas inmunocomprometidas (8).

Hacia el 2004, se evaluó la calidad del agua de los equipos odontológicos del Centro de Clínicas Odontológicas de la Universidad de Talca en México, en donde se evidenció que existe una amplia distribución de la contaminación microbiana, en concentraciones que superan lo establecido como normal y se aislaron principalmente Alcaligenes spp, Bacillus subtilis $\mathrm{y}$ Micrococcus spp, todos tienen como fuente probable el agua de la red, el primero es oportunista y los otros de baja patogenicidad (34). En el año 2007 se realizó un análisis microbiológico en unidades odontológicas de la Universidad Bornova-Izmir en Turquía, que demostró que las principales bacterias presentes en las muestras fueron: Pseudomonas spp, Mycobacterium spp, Staphylococcus spp, Legionella pneumophila. Estos microorganismos han sido descritos en pacientes inmunocomprometidos principalmente (35). En estudios más recientes, realizados en Italia, se encontró que en el agua de las unidades se obtuvieron recuentos de $24 \mathrm{X} 104 \mathrm{UFC} / \mathrm{mL}$ (36).

Las bacterias Gram negativas presentan en la envoltura de la membrana celular los lipopolisacáridos, que juegan un papel importante en la patogénesis de las infecciones bacterianas, un estudio de la Universidad de British Columbia reportó la presencia de lipopolisacaridos bacterianos en muestras de agua tomada de la unidad odontológica, al inicio de la actividad se encontraron 2.560 unidades de endotoxina por $\mathrm{mL}(\mathrm{UE} / \mathrm{mL})$, mediante lavado durante un minuto disminuyó a $800 \mathrm{UE} / \mathrm{mL}$, sin embargo después de lavados durante 5 a 10 minutos los niveles no llegaron a cero. Esto confirma la presencia de contaminación 
bacteriana por bacterias Gram negativas como Salmonella y E. coli y ratifica la necesidad de un buen manejo del agua de la unidad para evitar riesgo en el paciente (37).

Las bacterias que frecuentemente se encuentran en el agua de las unidades odontológicas son: Enterococcus spp., Achromobacter xyloxidans, Acinetobacter spp., Alcaligenes denitrificans, Bacillus spp., Bacillus subtitis, Enterobacterias, Flavobacterium spp., Klebsiella pneumoniae, Legionella pneumophila, Legionella spp., Methylobacterium mesophilica, Lactobacillus spp., Micrococcus luteus, Moraxella spp., Pasteurella spp., Pseudomonas aeruginosa, Burkholderia cepacia, Staphylococcus spp, Serratia marcescens, Streptococcus s.p., Xanthomonas spp, Mycobacterium gordonae, Ochromobacterium anthropi, Veillonella alkalescens, entre otras $(8,38)$.

Psendomonas aeruginosa y la Burkholderia cepacia son habitantes comunes del suelo y de las aguas naturales, pueden sobrevivir e incluso multiplicarse en aguas con muy bajo contenido de nutrientes. No es inusual entonces encontrar especies de Psendomonas en casi todos los abastecimientos de agua doméstica, en los tanques de almacenamiento o en los conductos de drenaje, debido a que los parámetros de control microbiológico para el agua de consumo humano, no garantizan la ausencia de este patógeno oportunista, que puede alcanzar recuentos potencialmente peligrosos para el ser humano y generar un elevado riesgo de infección cruzada en el ambiente odontológico (39).Un informe de Gran Bretaña ratificó a P. aeruginosa como la causa de las infecciones orales en dos pacientes dentales comprometidos médicamente. Burkholderia cepacia ha sido un importante patógeno respiratorio en los pacientes con fibrosis quística. Estas dos especies bacterianas tienen un alto grado de resistencia a los antimicrobianos y a las sustancias desinfectantes (40).

Legionella pneumophila y otras especies de Legionella han sido detectadas en el agua de las unidades odontológicas. L. pneumophila se descubrió en el agua de alrededor de 42 unidades en 35 lugares de práctica en Austria, en 3 de cada 5 unidades en una clínica hospitalaria dental de Londres, en el 4\% de las 194 unidades odontológicas en niveles por encima de los $100 \mathrm{UFC} / \mathrm{mL}$ de un hospital londinense para la enseñanza, y en varias unidades en la Universidad de Dresden, Alemania (41).

En los Estados Unidos L. pneumophila ha sido detectada en el agua de las unidades de una clínica dental escolar de Ohio y en el $8 \%$ de las muestras de agua tomadas de 28 instalaciones dentales en California, Massachussets, Michigan, Minnesota, Oregón y Washington. En la última investigación, nunca se detectó L. pneumophila en concentraciones por encima de los $1.000 \mathrm{UFC} / \mathrm{mL}$, pero otras especies de Legionella se descubrieron en el $68 \%$ de las muestras de agua probadas y en recuentos de al menos 10 .0oo UFC/mL en el $19 \%$ de las muestras (42).

Los géneros Acinetobacter, Alcaligenes, KlebsieIla y Serratia son bacilos Gram negativos, causantes de infecciones oportunistas en personas inmunocomprometidas y se encuentran presentes en el agua de las unidades odontológicas. Las bacterias orales de los géneros Bacteroides, Fusobacterium, Lactobacillus, Peptostreptococcus y Streptococcus están implicadas en las caries dentales y en la enfermedad periodontal, también 
son oportunistas cuando se acumulan en la placa o en las superficies de los dientes y han sido aisladas del agua de unidades odontológicas (8).

\section{Otros agentes infecciosos}

Aunque las bacterias son los agentes mas estudiados en el agua de las unidades odontológicas, se han reportado otros agentes infecciosos como priones, virus, hongos y protozoos (43 - 44). En las piezas de mano y líneas de agua de la unidad odontológica, debido a la retracción de fluidos orales, se han encontrado priones y virus como VIH, hepatitis B y herpes simplex (45). En otro estudio realizado en 2008, se demostró que las líneas de agua y las piezas de mano pueden ser contaminadas por agentes virales y son vehículo de infecciones cruzadas (46).

En un estudio llevado a cabo en Polonia, con el fin de determinar la presencia de hongos, se tomaron muestras del agua de la pieza de alta velocidad y de la pared de la línea de agua que conecta el reservorio de la unidad y las piezas de mano, los hongos identificados fueron: Aspergillus amstelodami, Aspergillus fumigatus, Aspergillus glaucus group, Aspergillus repens, Citromyces spp., Geotrichum candidum, Penicillium aspergilliforme, Penicillium pusillum, Penicillium turolense, Sclerotium sclerotiorum y Candida albicans, Candida curvata y otras levaduras (47). Un estudio similar se realizó en Turquía, en este se evaluaron 59 muestras de agua de las cuales 18 (30.5\%) fueron positivas para hongos identificados como Aspergillus flavus y Penicillium expansum (48). Algunas de las especies identificadas en los estudios anteriores, en ciertas circunstancias, especialmente en personas inmunosuprimidas, pueden causar infecciones oportunistas.
En el año 2006, se evaluó la influencia del protocolo de desinfección de las líneas de agua de las unidades odontológicas sobre la formación de bioaerosoles micóticos durante la consulta odontológica, en el estudio se determinó que la especie mas común fue Penicillium herquei seguido por Alternaria alternata, Penicillium roseopurpureum, Rhizopus nigricans, Aspergillus terreus, Geotrichum candidum, Aspergillus glaucus group, Cladosporium cladosporoides y PeniciIlium diversum. Los niveles de hongos totales reportados fueron más bajos después de la desinfección de las líneas de agua de las unidades, comparados con los obtenidos antes de la desinfección. Sin embargo, tanto antes como después de la desinfección prevalecieron las especies Penicillium herquei, Penicillium reseopurpureum y Alternaria alternata (49)

Respecto a los protozoos, se reportó en el año 2010 la detección de Acanthamoeba spp., en el 69\% de muestras de agua recolectadas antes y después de la consulta odontológica. No se ha demostrado la patogenicidad de esta ameba después de una contaminación oral o dental, pero su presencia puede ser una fuente de riesgo para pacientes en caso de tratamiento odontológico más profundo o inmunodepresión (50).

Debido a que las infecciones orales podrían ocurrir cuando los seres humanos y los microorganismos que contaminan el agua entran en contacto, todos los encargados de brindar atención en salud tienen la responsabilidad de reducir esta posibilidad de infección, particularmente cuando ésta se origina en una unidad odontológica. El mejoramiento de la calidad del agua en las unidades odontológicas es fundamental para el mantenimiento de la 
salud, para la alta calidad en la atención al paciente y para la protección del equipo de trabajo $(8,9)$.

\section{REFERENCIAS}

1. Nikaeen M. Microbial quality of water in dental unit waterlines. [citado 11 marzo de 2010]. www.blackwellpublishing.com.

2. Szymańska J, Dutkiewicz J. Concentration and species composition of aerobic and facultatively anaerobic bacteria released to the air of a dental operation area before and after disinfection of dental unit waterlines. Ann Agric Environ Med 2008;(15):301-7.

3. García N. Contaminación en los ductos de agua de las Unidades dentales. Ejercicio profesional y administración. [citado 11 de Febrero de 2011]. www.medilegis.com/ BancoConocimiento/O/Odontologica-v1n4ejercicio/ejercicio.htm.

4. Organización para la Seguridad y los Procedimientos de Asepsis (OSAP). Postura Sobre Las Líneas De Agua En La Unidad Dental. [citado 8 de agosto de 2010]. www.osap.org/ displaycommon.cfm?an=1\&subarticlenbr $=111$.

5. Barbeau J. Lawsuit Against a Dentist Related to Serious Ocular Infection Possibly Linked to Water from a Dental Handpiece. JCDA. [citado 02 diciembre de 2010]. http://cda-adc.ca/jcda/ vol-73/issue-7/618.html.

6. Yañez MA. Control de la contaminación microbiológica en unidades dentales. Gaceta dental. [citado 6 julio de 2010]. http://dialnet. unirioja.es/servlet/articulo?codigo $=1182821$.

7. Pankhurst CL, Coulter W, PhilpottHoward JN. Evaluation of the potential risk of occupational asthma in dentists exposed to contaminated dental unit waterlines 2005; Prim Dent Care 12: 53-9.

8. Milleri $\mathrm{CH}$. Los microbios en el agua de las unidades dentales. Rev Cubana Estomatol $1996 ; 33: 3$.

9. Acosta E, Maupomé G. Transmisión de las infecciones en el consultorio dental Rev Pract Odontol 1994; 15: 9-12.

10. Pankhurst CL, Philpott-Howard JN. The environmental risk factors associated with medical and dental equipment in the transmission of Burkholderia cepacea in cystic fibrosis patients. J Hosp Infect. 1998;(32): 249-255.

11. Rodríguez S, Shenoy $V$, Joseph $M$. Condiciones que favorecen la colonización bacteriana del agua en Unidades dentales. [citado 11 de Febrero de 2011]; www.siicsalud. com/dato/dat048/06410014.htm.

12. Smith A. Is the water in dental units clean? British Dental Journal. [citado 13 enero de 2011]. http://aurainfectioncontrol.co.uk/ Andrew1.pdf.

13. Muñoz J, Hernández D, Moreno A. Calidad bacteriológica del agua de una clínica odontológica rural de la facultad de odontología de la Universidad Autónoma de Zacatecas. Revista de la Asociación Dental Mexicana 2002; (59): 50-57.

14. Williams JF, Molinari JA, Andrews N. Contaminación microbiana de las líneas de agua de la unidad dental: orígenes y características. JADA 1996; 127:1198-1196.

15. Charles M, Christopher R, Martel B.S. How Does Time-Dependent Dental Unit Waterline Flushing Affect Planktonic Bacteria Levels?. Journal of Dental Education 2002;(66): 549-555

16. Chate RA. An audit. Improves the quality of water within the dental unit water lines of three separate facilities of a United Kingdom NHS Trust. Br Dent J 2006;(201): 565-569.

17. Petty S, Tarsitani G. Detection an Quantification of Dental Unit Water Line Contamination by Oral Streptococci infection control and hospital epidemiology [citado 6 septiembre de 2010]. http://cat.inist.fr/?aModel $\mathrm{e}=$ afficheN\&cpsidt $=17794635$.

18. Walter JT, Bradshaw DJ, Bennett AM. Microbiol biofilm formation and contamination of dental- unit water systems in general dental practice. Appl Environ Microbiol 2000; 66: 3363-3367.

19. Forde A, O'Reilly P, Fitzgerald G. Microbial contamination of dental unit water systems. J Ir Dent Assoc 2005;(51):115-8.

20. Szymanska J, Wdowiak L, Puaez E, Stojek N. Microbial quality of water in dental unit reservoirs. Ann Agric Environ Med 2004; 11:355-358.

21. Szymanska J. Control methods of the microbial water quality in dental unit waterlines. Ann Agric Environ Med 2003;(10):1-4. 
22. Smith AJ, McHung S, McCormick L. A crosssectional study of water lines in dental practices in the West of Scotland. $\mathrm{Br}$ Dent J 2002; 52: 192-205

23. Wallace JR, Swenson JM, Silcox VA. Spectrum of disease due to rapidly growing Mycobacteria. Rev Infect Dis 1983;(5): 657-777.

24. Schulze-Robbecke R, Feldman C, Fischeder R. Dental units: an environmental study of sources of potencially pathogenic mycobacteria. Tuber Lung Dis 1995; 76: 318-323.

25. Meiller TF, Depaola LG, Kelley JI. Dental unit waterlines: biofilms, disinfection and recurrence. J Am Dent Assoc 1999; 130:65-72.

26. Liaqat I, Sabri N. Biofilm, dental unit water line and its control. Afr. J. CIn. Exper. Microbiol 2010; (12): 15-21

27. Tanahashi T, Tonami K, Kurosaki N. Effect of a small electric current on sterilization of a dental unit water line. J med dent sci 2006;(53):111-118

28. Walker RJ, Burke FJ, Miller $\mathrm{CH}$, Palenik CJ. An investigation of the microbial contamination of dental unit air and water lines. Int Dent J $2004 ; 54: 438-44$.

29. Anonymus. Recommended infectioncontrol practices in dentistry. MMWR. 1993;(42): 1-11.

30. Mills SE, LLauderdale PW. Reducción de la contaminación microbiana en las unidades dentales con iodo-povidona al 10\%. JADA 1986; 113:280-284.

31. Gross A, Devine MJ and Cutright DE. Microbial contamination of dental units and ultrasonic scalers. Periodontol 1976;(47): 670-3.

32. The SD, Vant Hof MA. Bacterial Conditions of Water in Dental Units. J Dent Res 1975; 54: 1064-68.

33. Bagga BS, Murphy RA. Contamination of dental unit cooling water with oral microorganisms and its prevention. JADA 1984;109: 712-16.

34. Arraigada A. Control de Infección en los Ductos de Equipos Dentales de las Clínicas Odontológicas de la Universidad de Talca. Revista Dental de Chile. [citado 6 agosto de 2010]. http://dspace.utalca.cl/retrieve/7371/ arriagada_vergara.pdf.
35. Uzel A. Microbiological evaluation and antibiotic susceptibility of dental unit water systems in general dental practice. Int J Dent Hygiene 2008; 6-10.

36. Pasquarella C, Veronesi L, Castiglia P, Liguori G. Italian multicentre study on microbial environmental contamination in dental clinics: a pilot study. Sci Total Environ 2010; 408:4045-51.

37. Putnins EE, Di Giovanni D, Bhullar AS. Dental unit waterline contamination and its possible implications during periodontal surgery. Periodontol 2001; 72:393-400.

38. Liaqat I. Fourier transform infrared spectroscopy of dental unit water line biofilm bacteria. Spectroscopy 2009; 3: 175-189.

39. Chacón Ch, Isvelia M, Yépez G, Castillo C. Aislamiento de especies de Pseudomonas de las líneas de agua de las unidades odontológicas. [citado febrero 17 de 2011] www.actaodontologica. com/ediciones/2010/1/art1.asp

40. Walker JT, Bradshaw DJ, Fulford MR, Marsh PD. Microbiological evaluation of a range of disinfectant products to control mixed-species biofilm contamination in a laboratory model of a dental unit water system. Appl Environ Microbiol 2003; 69: 3327-3332.

41. Pankhurst CL, Coulter W, Philpott-Howard JN. Prevalence of Legionella pneumophila antibodies in general dental practitioners in London and rural Northern Ireland. $\mathrm{Br}$ Dent J 2003; 195: 591-594.

42. Challacombe SJ, Fernández LL. Detecting Legionella pneumophila in water systems: a comparison of various dental units. JADA 1995; 126: 603-608.

43. Santhosh K, Arjun S, Disha G. Microbial contamination in dental unit water lines (DUWL) in private dental clinics of Udaipur City, India. Int J Infect Control 2009; 5: 1-10

44. Szymańska J. Microbiological risk factors in dentistry. Current status of knowledge. Ann Agric Environ Med 2005; 12:157-63.

45. Stephen R. Prions and dentistry. J R Soc Med 2002; 95: 178-181

46. Artini M, Scoarughi GL, Papa R. Specific anti cross-infection measures may help to prevent viral contamination of dental unit waterlines: a pilot study. Infection 2008; 36:467-71. 
47. Szymańska J. Evaluation of mycological contamination of dental unit waterlines. Swed Dent J 2009; 33:161-72.

48. Göksay D, Cotuk A, Zeybek Z. Microbial contamination of dental unit waterlines in Istanbul, Turkey. Environ Monit Assess 2008; 147:265-9.
49. Szymańska J. Exposure to airborne fungi during conservative dental treatment. Prim Dent Care 2005; 12:53-9.

50. Trabelsi H, Sellami A, Dendena F. Freeliving Amoebae (FLA): morphological and molecular identification of Acanthamoeba in dental unit water. Parasite 2010; 17:67-70. 